\title{
AN OTA-BASED DIGITAL-TO-ANALOG CONVERTER
}

\author{
A.R. AL-ALI, M.T. ABUELMA'ATTI and A. SHABRA \\ King Fahd University of Petroleum and Minerals, Box 203 Dhahran 31261 Saudi Arabia \\ (Received November 14, 1993; in final form December 15, 1993)
}

\begin{abstract}
A simple circuit for implementing a current-switching digital-to-analog converter is presented. The circuit uses operational transconductance amplifiers (OTAs) for switching a number of equal-value current sources. An R-2R ladder attenuator is used to scale the currents in a binary form. Experimental results show good performance.
\end{abstract}

\section{INTRODUCTION}

Digital-to-analog conversion can be accomplished using various techniques. In most applications, and particularly for IC implementation, current switching is normally preferred over voltage switching. This is because during current switching the network node-voltages remain unchanged. This minimizes the switching transients and the corresponding settling time. On the other hand, some of the inherent problems, such as emitter area scaling or unequal switching associated with binaryweighted current sources, can be avoided by using equal-value current sources in conjunction with an R-2R ladder, which is used as an attenuator to scale currents in a binary manner ${ }^{1}$. Therefore, digital-to-analog converter implementations using current switching of equal-value current sources in conjunction with low values of resistances in the $R-2 R$ ladder are attractive.

The performance characteristics and the response speed of current-switching digital-to-analog converters depend strongly on the characteristics of the current switches used in the circuit. A suitable current switch must have a) high speed, b) good buffering, c) low reverse leakage, and d) logic compatability. The operational transconductance amplifier (OTA) is essentially a high input impedance, high output impedance voltage-controlled current source that produces an output current proportional to the differential input voltage. The transconductance of the OTA is proportional to the auxiliary bias current $\mathrm{I}_{\mathrm{ABC}}$. Differential input voltage imbalances at the OTA inputs greater than tens of millivolts are sufficient to cause all the bias current $\mathrm{I}_{\mathrm{ABC}}$ to be either pushed or pulled from the output lead. In addition, measurements show that the output current reverses its direction very quickly in response to changes in differential input polarity. For example, with $\mathrm{I}_{\mathrm{ABC}}=50 \mu \mathrm{A}$ and differential input voltage $= \pm 2 \mathrm{~V}$, the output current completely reverses its direction in $150 \mathrm{~ns}$, while for $\mathrm{I}_{\mathrm{ABC}}>200 \mu \mathrm{A}$, the reversal occurs in less than 50 $\mathrm{ns}^{2}$. It is, therefore, reasonable to assume that the output current switches instantaneously from one polarity to the other. It appears, therefore, that the OTA meets 


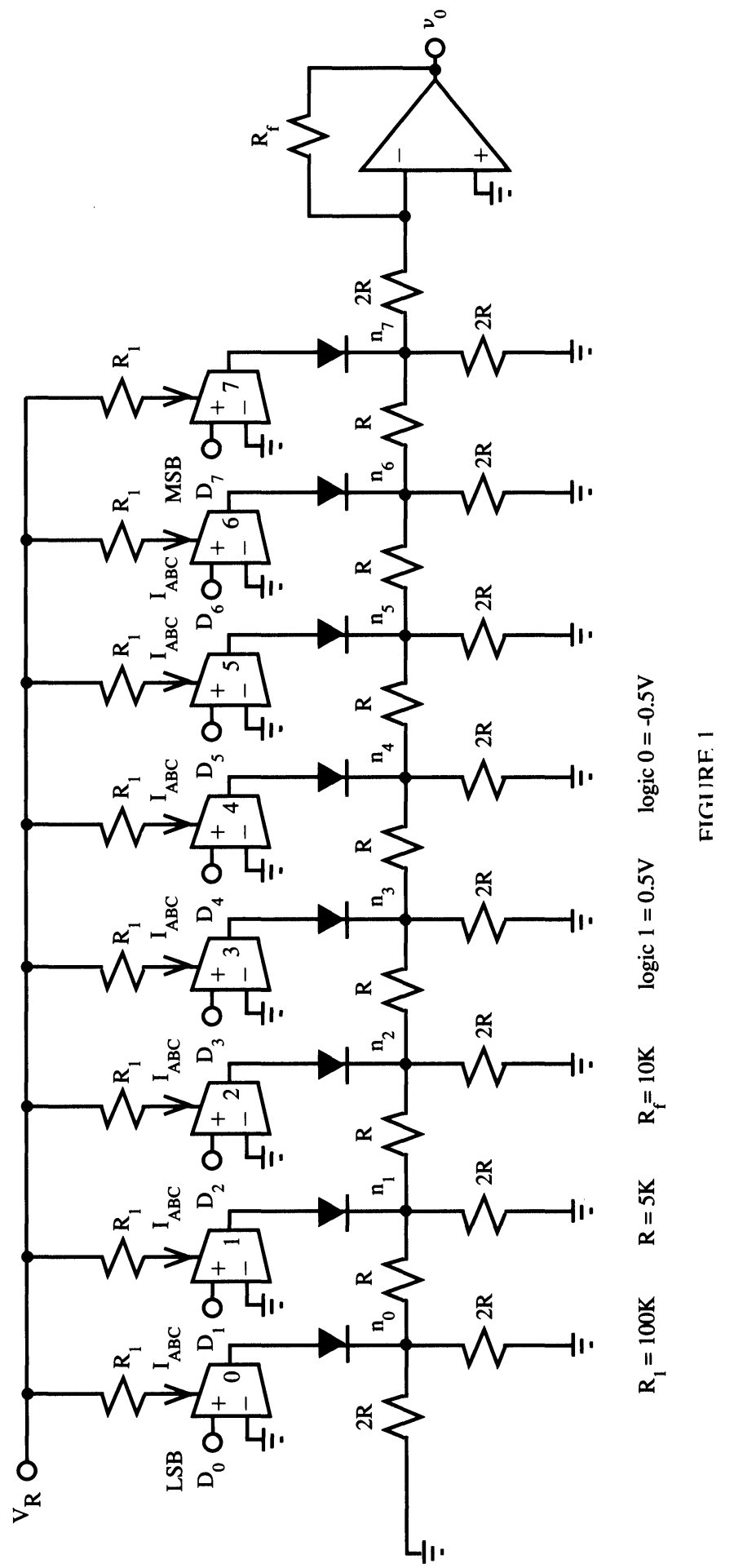


all the requirements of a high-performance current switch. Nevertheless, the logic compatibility requirements often necessitates the inclusion of de level-shifting functions within the switch. This can be easily achieved.

The major intention of this paper is to investigate the feasibility of designing OTA-based digital-to-analog converter using current switching of equal-value current sources in conjunction with an R-2R ladder attenuator to scale the currents in a binary manner.

\section{PROPOSED CIRCUIT}

The proposed circuit is shown in Fig. 1. The circuit uses one OTA-based current switch for each additional input bit. The diode at the output lead of the OTA ensures that the $R-2 R$ ladder node $n_{k}$ receives current only when the $k$ th OTA digital input is at logic 1. The circuit was constructed using the CA3080 OTA. This OTA is not a perfect one. Its output current magnitude is not exactly equal to $\mathrm{I}_{\mathrm{ABC}}$ but is slightly less than it. Moreover, the difference between these two currents is not fixed but is a function of $\mathrm{I}_{\mathrm{ABC}}$. As $\mathrm{I}_{\mathrm{ABC}}$, increases the percentage difference between it and the output current also increases. Furthermore, the base-to-emitter voltage $\mathrm{V}_{\mathrm{BE}}$ of the OTA is dependent on $\mathrm{I}_{\mathrm{ABC}}$. This complicates the injection of a current into the junction using a voltage.

Despite these nonideal characteristics it was found that the maximum error was less than $\pm \frac{1}{2} \mathrm{LSB}$. In addition, the circuit exhibited no offset error and was monotonic over the entire range of operation. Moreover, the possible gain error of the circuit can be compensated by adjusting the reference voltage $V_{R}$. It must be pointed out that no particular attention was paid to matching the OTAs. This might suggest that higher accuracy and higher bit numbers can be realized using this design approach. Also, the influence of temperature can be compensated by using temperature compensated OTAs ${ }^{3.4}$.

\section{CONCLUSION}

In this paper, a novel implementation for a digital-to-analog converter has been presented. The proposed circuit, shown in Fig. 1, uses OTAs for the switching of a number of equal-value current sources in conjunction with an R-2R ladder attenuator to scale the currents in a binary manner. Although the experimental results show good performance, improved accuracies can be achieved by using matched temperature compensated OTAs and high precision R-2R ladder networks.

\section{REFERENCES}

1. A.B. Grebene, Bipolar and MOS Analog Integrated Circuit Design, John-Wiley \& Sons: N.Y., 1984.

2. J.W. Haslett, Current-switching linear VCO, Electronics Letters, Vol. 25, 1989, pp. 139-140.

3. H.S. Malvar and M. Luettgen, Temperature compensation of OTA-based filters and amplifiers, Electronics Letters, Vol. 23, 1987, pp. 890-891.

4. M.T. Ahmed, I.A. Khan and T. Parveen, Wide range electronically tunable component multipliers, International Journal of Electronics, Vol. 65, 1988, pp. 1007-1011. 

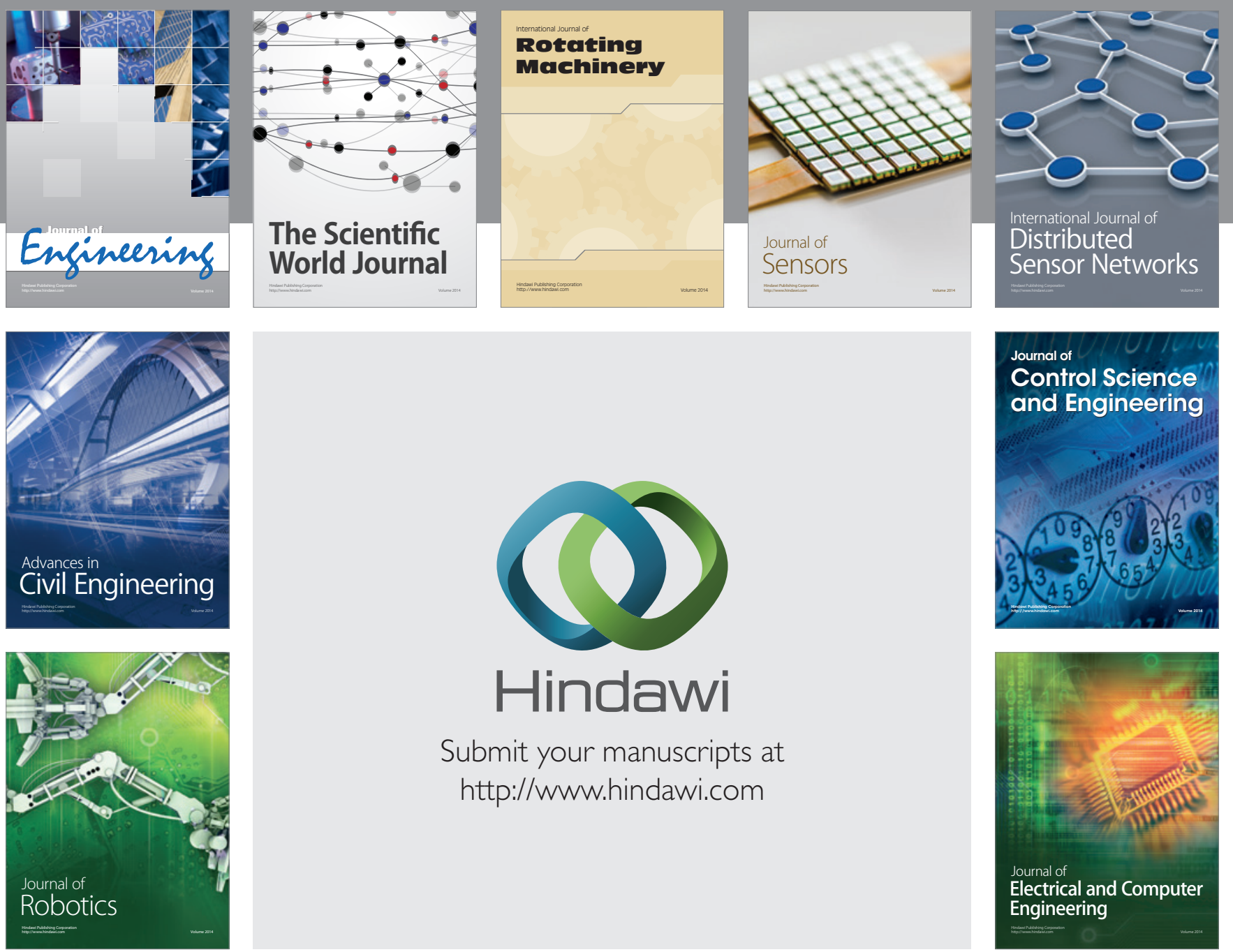

Submit your manuscripts at

http://www.hindawi.com
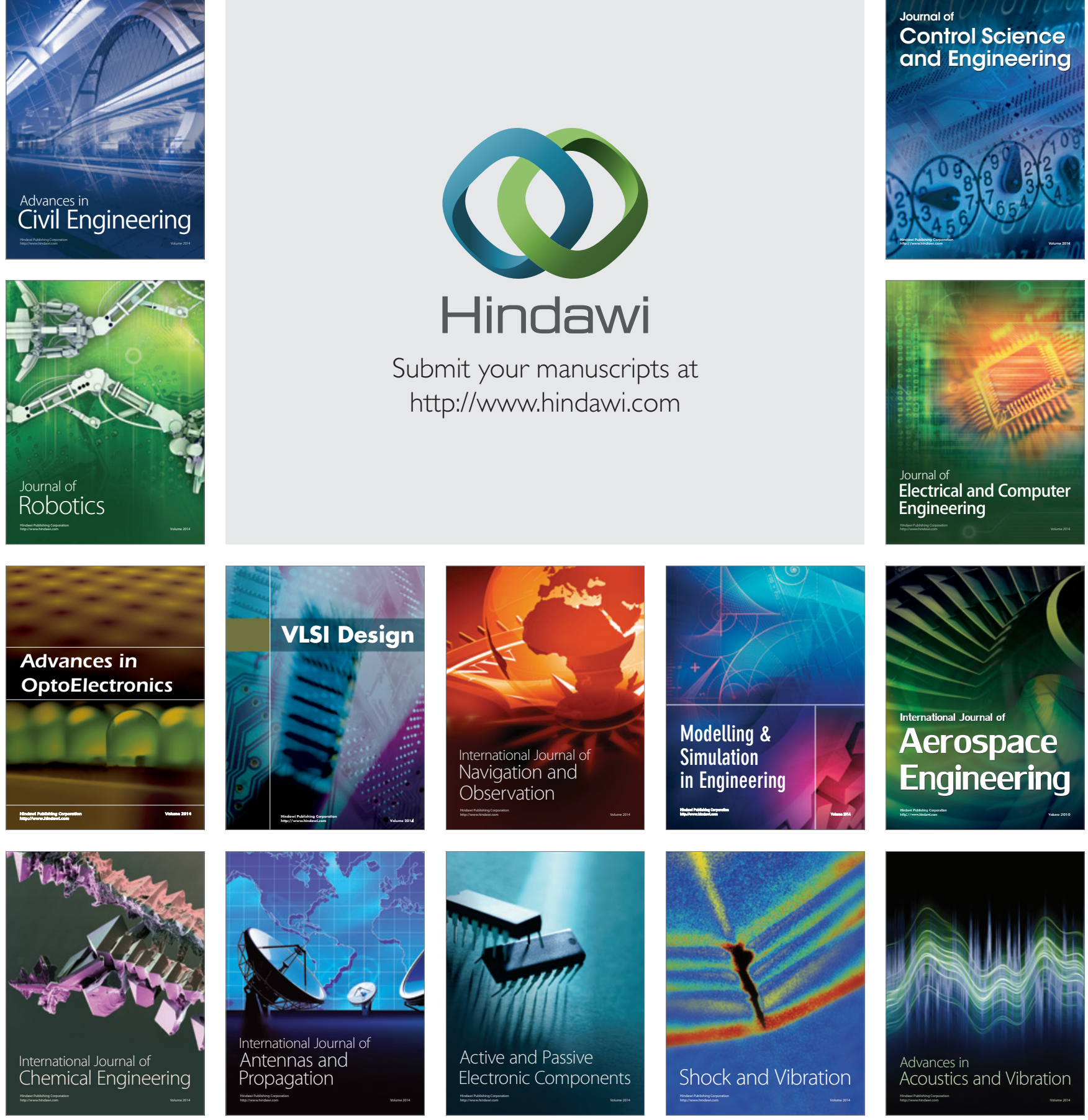